\title{
Nanostructure formation in Silicon photovoltaic cell surface by femtosecond laser pulses
}

\author{
K. A. Al-Naimee \\ Department of Physics, College of Science, University of Baghdad, Baghdad, Iraq \\ CNR- Istituto Nazionale di Ottica Applicata, Largo E. Fermi 6, 50125, Florence, Italy \\ kais.al-naimee@inoa.it
}

\begin{abstract}
The experimental evidence of the effect of femtosecond laser pulses on the spectral response of a Silicon photovoltaic cell is demonstrated and investigated. The response of this device is covering the visible to near infrared spectral region. The responsivity of the photovoltaic cell is enhanced from $0.18 \mathrm{~A} / \mathrm{W}(0.5 \mathrm{~A} / \mathrm{W}$ to $0.85 \mathrm{~A} / \mathrm{W})$ to $0.25 \mathrm{~A} / \mathrm{W}$, this means that the conversion efficiency increases from about $9 \%$ to about $14 \%$ due to irradiation effect. All treatments and measurements have been done at room temperature. The observed enhancement is related to the appearing of nano-structured groves in the $700-900 \mathrm{~nm}$ range.
\end{abstract}

Keywords: Silicon photovoltaic cell, nanostructure, femtosecond laser pulse, irradiation, infrared

\section{Introduction}

Silicon is the most commonly used semiconductor in optoelectronic devices and Silicon photodiodes are extensively used in industrial applications as reliable devices for light to electricity conversion. These features are especially important in the field of optical radiometry in which measurements of photometric and radiometric quantities have to be done with a high level accuracy. The description of high accuracy interpolation of the quantum yield of silicon photodiodes (detectors) in the near UV was reported by Kübarsepp et al. (2000). They also compared the results of the quantum yield calculations and measurements obtained using of Silicon trap detector. Since 1969 the amorphous Silicon plays a crucial role in producing low cost-effective solar cells. However, the photovoltaic cells made of this material tend to have lower energy conversion efficiency than bulk silicon. Carey et al (2005) investigated the I-V characteristics and responsivity of photodiodes fabricated micro structured Silicon by using femtosecond laser pulses in a Sulfur containing atmosphere. Silicon surfaces irradiated with high intensity nanosecond laser pulses in the environment of Sulfur-containing gases have absorption near unity from near UV $(250 \mathrm{~nm})$ to NIR $(2500 \mathrm{~nm})$ at photon energies well below the band gap of ordinary Silicon (Crouch et al., 2004). Spontaneously developed micro structures on Silicon surfaces under the effect of short laser pulses irradiated in different ambient atmospheres have been reported (Xiaoyun et al. 2005; Myers et al. 2006). The experimental results reveal that the ambient atmosphere and the laser pulse duration play key roles on the formation of microstructures.
The physical processes responsible for the periodic structure formation in femtosecond laser ablation of thin films surfaces have been reported (Miyaji \& Miyazaki, 2008). It has been found that an initial random distribution of nanoscale ablation traces is periodically structured with an increase in superimposed laser pulses.

In the present work, the formation of nanostructures on the irradiated photovoltaic surface is observed and the experimental procedure to enhance the efficiency of a bare silicon photovoltaic cell from $9 \%$ to $14 \%$ is reported.

\section{Experimental procedure}

The experimental procedure is done by two steps, the first step is the Photovoltaic cell (sample) irradiation by femtosecond laser pulses, and the second step is the response measurement of the sample. The setup of the photovoltaic sample irradiation is depicted in Fig.1. The

Fig. 1. Schematic diagram of the experimental setup of sample irradiation

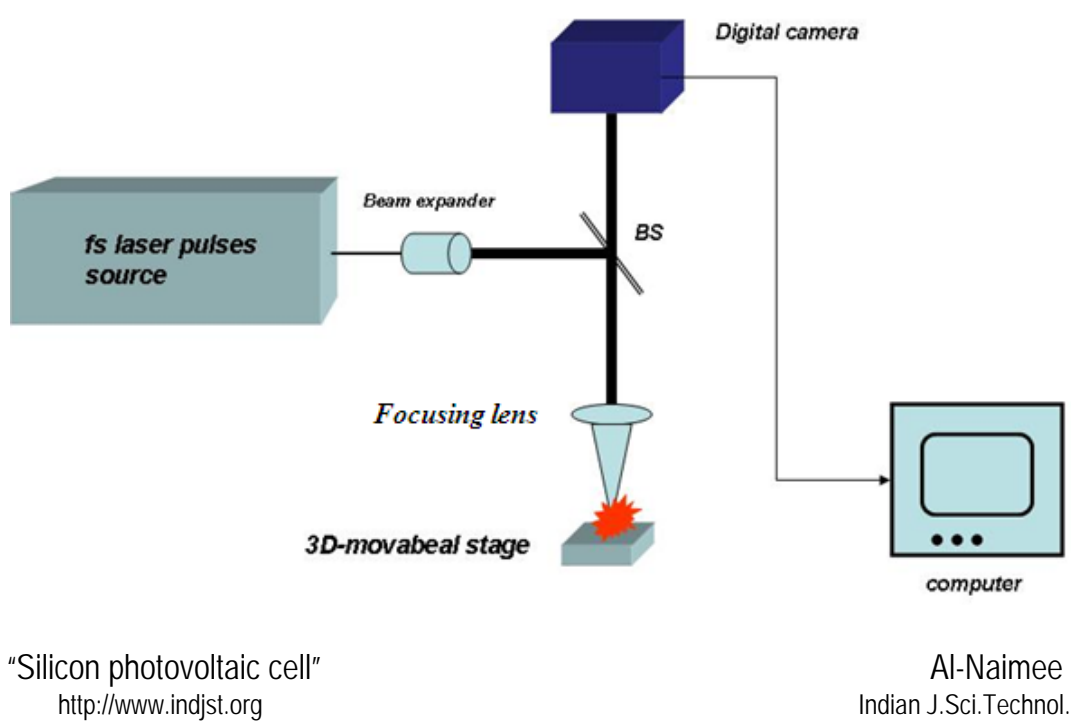


sample was irradiated by a tunable mode-locked Ti:sapphire laser with $100 \mathrm{fs}$ pulse duration, $80 \mathrm{MHz}$

Fig. 2. The periodic structured of the irradiated cell, by a $100 f$ laser pulses, $800 \mathrm{~nm}$ wavelength, the figure shows $12 \mu \mathrm{m}$ length

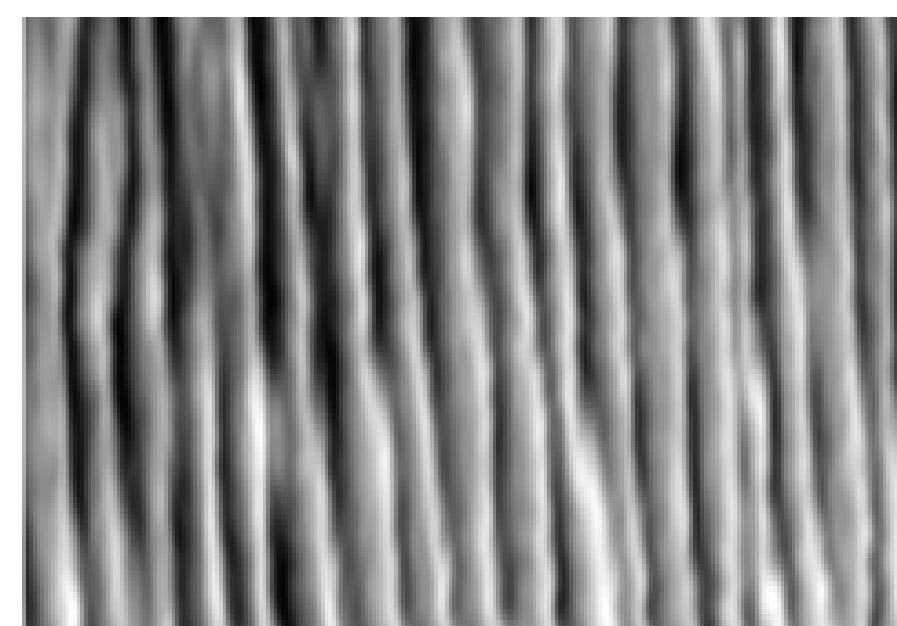

frequency at $800 \mathrm{~nm}$ wavelength of operation. During the irradiation process a focusing objective with high numerical aperture was used in air environment. The laser beam has been expanded by a $1 \times 4$ beam expander and then focused on the target by means of a $100 \mathrm{~mm}$ focal length lens. The irradiating beam was directed normal to the anode side of the sample. A digital camera was utilized to observe the sample movement during the irradiation of the cell. The irradiation process created numerous defect sites and modified the sample surface. The time responses of the irradiated and unirradiated samples have been measured by a $500 \mathrm{MHz}$ oscilloscope. A scanning Electron Microscope (SEM) images of the structures was obtained in air environment. Line scan was performed at a fixed scan speed.

The spectral response of the samples was measured by standard method using computerized monochromatic before and after the irradiation process. The system instrumentation was controlled by a PC via the RS232 interface. The output of the monochromator was directed to two output windows. One beam was directed to a calibrated detector and the other to the sample under test. During the system operation a broad band light source was passed through a monochromator signaling the desired frequency of the light illuminating the photodiode. The photovoltaic device current was measured by means of the semiconductor parameter analyzer and the results were stored on the PC. The measurements were then repeated in the (400-900) nm wavelength range.

Results and discussion (nanoscale surface ripple formation and response measurements)

A semi periodic structure (lines) was formed and observed in the range of $700-900 \mathrm{~nm}$. The SEM images show a semi periodic structure known as ripples or grooves in the submicrometer range (Fig.2). Under laser pulses, the surface becomes highly corrugated nanostructure with valley. The spacing of these nanochannels decreases as the number of laser pulses increases and fluence. The formation of the nano-ripple is attributed to the optical interference of the incident and scattered laser pulses from the sample surface. This approach leads to a new generation of photovoltaic cells. Indeed, it enhances the photovoltaic material responsivity and hence the conversion efficiency. Since the efficiency of the amorphous Silicon photovoltaic cells is increased from $7 \%$ to about $9 \%$ during the last 40 years utilizing

Fig. 3. Spectral response of the irradiated and unirradiated photovoltaic solar cells (a) and comparison of the spectral response curve of irradiated and un irradiated cells (b)
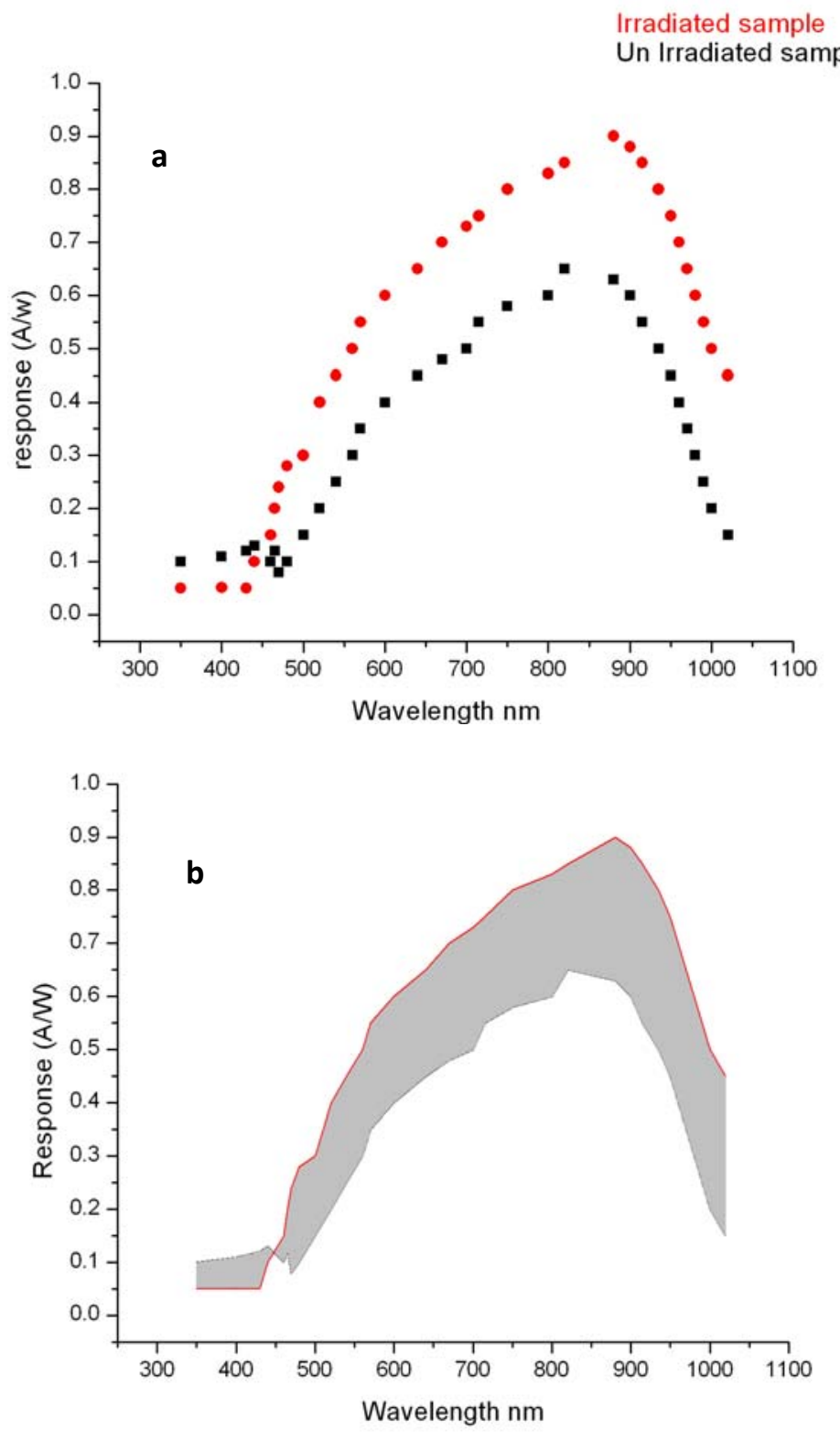

Research article

CIndian Society for Education and Environment (iSee)
"Silicon photovoltaic cell" http://www.indjst.org
Al-Naimee Indian J.Sci.Technol. 
different methods, the stepping to $14 \%$ efficiency must be by only rearranging the micro crystal structure of the cell surface. enhance the responsivity and the conversion efficiency of the photovoltaic cell. Ultrashort laser pulses should be still economically reasonable in a large scale production.
Fig. 4. The gain response of the irradiated sample at two different wavelengths

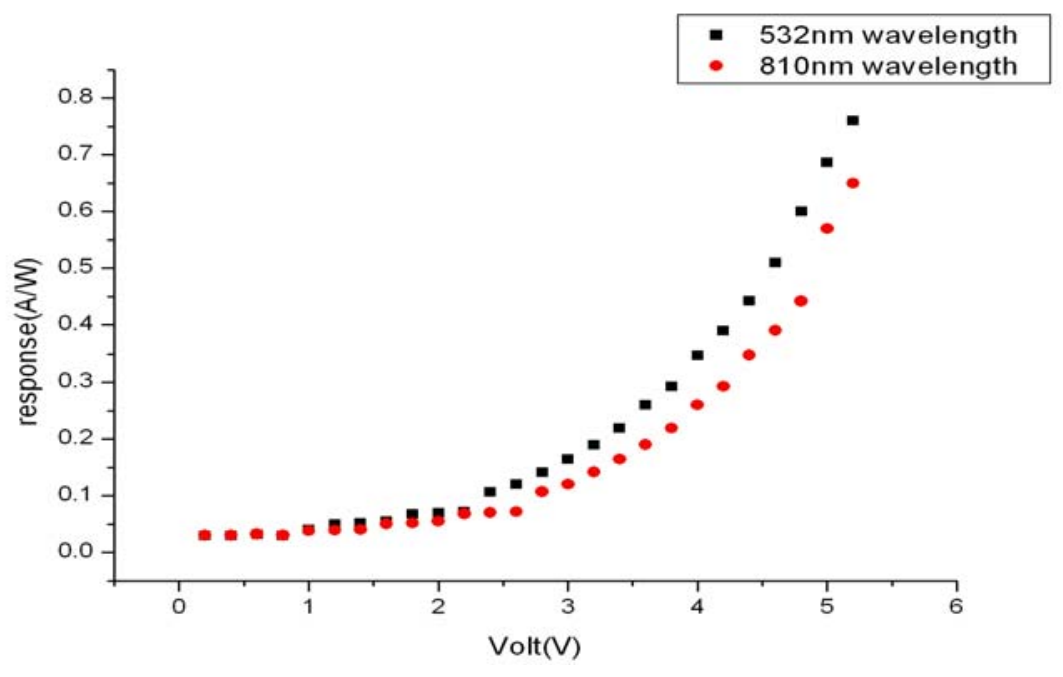

The effect of the irradiation of ultrashort laser pulses on the spectral response is shown in Fig. 3a. It reveals the dependence of the responsivity versus wavelength and the role of the laser effects at room temperature. The relative response increased after the irradiation processes. The efficiency of the irradiated sample is enhanced compared to unirradiated sample at wavelengths longer than $500 \mathrm{~nm}$. Hence, the responsivity of the photovoltaic cell is increased from $0.18 \mathrm{~A} / \mathrm{W}$ to $0.25 \mathrm{~A} / \mathrm{W}$ due to irradiation effect.

The conversion efficiency of the irradiated cell is calculated using the well known formula

$$
\eta=I \mathrm{~V} / \mathrm{Al} \text { 。 }
$$

where $I$ is the value of the current, $V$ is the voltage, $I_{0}$ is the solar intensity and $A$ is the solar cell area. The results show that the measured efficiency for the bare Silicon photovoltaic cell increases from $9 \%$ before irradiation to $14 \%$ after. However, the area under the response curves as shown in Fig. $(3, b)$ indicating the total response of the irradiated samples. The ratio of the total quantum yield, before and after irradiation, increases by a factor 1.43 over the whole spectral range. Finally, we address the question: how met an irradiation depends on the laser wavelength. The gain response curves as a function of the bias voltage for samples irradiated at different wavelengths are shown in Fig.4. The trend is the same at different wavelengths, $532 \mathrm{~nm}$ and $810 \mathrm{~nm}$.

\section{Conclusions}

We have demonstrated how sub-microstructures can be created in photovoltaic cell by irradiating the sample with femtosecond laser pulses. This technique helps to

\section{Acknowledgment}

Author acknowledges M. Bellini and A. Zavatta for their help during the irradiation the samples and ICTP for the financial support in the framework of the TRIL program.

\section{References}

1. Carey JE, Crouch $\mathrm{CH}$, Shen $\mathrm{M}$ and Mazur E (2005) Visible and near-infrared responsivity of femtosecond-laser microstructured silicon photodiodes. Opt. Lett. 30, 1773-.

2. Crouch $\mathrm{CH}$, Carey JE, Warrender JM, Aziz MJ, Mazur E and Génin FY (2004) Comparison of structure and properties of femtosecond and nanosecond laserstructured silicon. Appl. Phys. Lett. 84, 1850-

3. K"ubarsepp T, K"arh"a P, and Ikonen E (2000) Interpolation of spectral responsivity of Silicon photodetectors in the near UV. Appl. Optics. 39, 915.39,1.

4. Miyaji G and Miyazaki K (2008) Origin of periodicity in nanostructuring on thin film surfaces ablated with femtosecond laser pulses. Optics Express. 16, 20, 16265.

5. Myers A, Farrell R, Karger A, Carey E and Mazur E (2006) Enhancing near-infrared avalanche photodiode performance by femtosecond laser microstructuring. Appl.Opt. 45, 35, 8825.

6. Xiaoyun H, Jun Fan, Ting Li, Dekai Zhang, Jintao Bai and Xun Hou (2005) Influence of preparation technique on the microscopic structure and surface morphology of nanometer $\mathrm{TiO}_{2}$ thin films. Proc. of the SPIE. 5635, 390-398. 